\title{
Entretien avec Régis Campo, un compositeur « ludique »
}

Christophe Franco-Rogelio et Jean-Michel Denizart

\section{(2) OpenEdition \\ Journals}

Édition électronique

URL : http://journals.openedition.org/lcc/956

DOI : $10.4000 /$ lcc. 956

ISSN : 2430-4247

Éditeur

Université Aix-Marseille (AMU)

Référence électronique

Christophe Franco-Rogelio et Jean-Michel Denizart, « Entretien avec Régis Campo, un compositeur " ludique » », Les chantiers de la création [En ligne], 7 | 2014, mis en ligne le 24 janvier 2015, consulté le 08 avril 2020. URL : http://journals.openedition.org/lcc/956; DOI : https://doi.org/10.4000/lcc.956

Ce document a été généré automatiquement le 8 avril 2020.

Tous droits réservés 


\title{
Entretien avec Régis Campo, un compositeur « ludique »
}

\author{
Christophe Franco-Rogelio et Jean-Michel Denizart
}

\section{NOTE DE L'ÉDITEUR}

Cet entretien a été réalisé le 28 mai 2014 à l'Université d'Aix-Marseille, à Aix-enProvence. La revue Les Chantiers de la Création, anciennement nommée e-lla, a convié Régis Campo pour une discussion autour son activité de compositeur, menée par Christophe Franco-Rogelio et Jean-Michel Denizart, doctorants et membres de la revue. Régis Campo a commencé ses études de composition à Marseille au Conservatoire National Régional (CNR) - dans lequel il est actuellement professeur -, dans la classe de George Bœuf auquel il a succédé. Il a également étudié à la Faculté des lettres d'Aix-enProvence en licence de philosophie, et au CNSM de Paris dans la classe de Gérard Grisey, compositeur dit de l'école spectrale, un grand nom de la musique du XXe siècle. Premier prix de composition à la sortie du CNSM, vous allez rencontrer Edison Denisov et Henri Dutilleux, grands compositeurs également. Suit une succession de prix, le prix Gaudeamus, le prix Dutilleux ... Le catalogue de composition de Régis Campo est assez large, il englobe notamment beaucoup d'œuvres orchestrales, ce qui est notable, des œuvres pour effectifs plus réduits, pour solistes également et de nombreuses œuvres vocales dont Les Cris de Marseille, titre inspiré des Cris de Paris de Clément Janequin.

\section{Entretien}

Christophe fRANCO-ROgelio : Les commentateurs de votre œuvre emploient souvent les termes second degré, atypique et ludique, qui est l'adjectif le plus récurrent. Et c'est vrai que lorsqu'on découvre une œuvre comme Wow! et qu'on la compare à des œuvres d'avantgarde des années 50, par exemple de Boulez pour ne pas le citer, dont l'esthétique est un peu austère, on peut se dire que le terme ludique est plutôt adéquat. 
Au-delà de l'étiquette, comment expliquez-vous ce point de vue ?

Régis Campo: Lorsque j'ai terminé mes études musicales dans les année 90 au Conservatoire de Paris, j'ai senti, et c'est commun dans l'art dit d'avant-garde, un certain académisme du sérieux et une dogmatisme certain. Il est évident que l'on ne pouvait plus créer d'une manière similaire avant et après la guerre. Bien entendu, cette nouvelle avant-garde était absolument naturelle après guerre puisqu'il fallait partir sur d'autres bases. Il y avait un croisement violent entre plusieurs générations et je comprends très bien les jeunes loups des années 50 qui ont voulu balayer un passé, phénomène similaire dans le cinéma avec la Nouvelle Vague par exemple. Le compositeur allemand Helmut Lachenmann a dit qu'il semblait écrire à partir d'un champ de cendres, en un sens je le comprends aussi. Le grand danger serait pour notre jeune génération (disons celle des années 70) de devenir amnésique et de balayer à notre tour ce qui s'est passé dans les années 50 , de faire table rase d'une table rase et de revenir en arrière!

Ma musique fut très vite connotée ludique. Je ne peux pas enlever cette étiquette, elle existe par défaut je dirais. Or ce terme de ludique a toujours existé, il est intrinsèque à toute musique. Les variations Goldberg de Bach sont ludiques par définition, Mozart et Stravinsky sont ludiques. Mais c'est par défaut que ce terme est arrivé, comme le terme malheureux de musique répétitive. Terme flou que l'on pourrait pourtant accoler au prélude en do de Bach ou à Vivaldi !

CFR: Etienne Kippelen, qui a été un de vos élèves au CNR de Marseille, et qui est actuellement professeur de composition en musicologie à l'université d'Aix-Marseille, a rédigé, il y a quelques années, un article dont le titre, si je me souviens bien, est « le ludisme dans la musique des années 2000 », publié dans L'art des année 2000 dirigé par Sylvie Coellier et Jacques Amblard. Dans cet article Etienne Kippelen décrit le ludisme très précisément comme étant un mouvement qui serait lié à un aspect très sombre que revêt la première décennie des années 2000, avec les attentats du 11 septembre etc. et il fait le lien avec d'autres compositeurs, dont certains plus anciens, notamment Ligeti, ses dernières pièces surtout. Mais il parle également de Philip Glass, d'Arvo Pärt et d'Eric Satie. Si les termes qui reviennent également le plus souvent sont ceux d'immédiateté et de simplicité d'écriture, je vous demanderais si ces notions vous correspondent et si vous vous sentez par conséquent proche esthétiquement de compositeurs comme Ligeti ou Satie?

RC: Ligeti est un compositeur phare pour toutes les générations et esthétiques confondues. Côtoyant les compositeurs d'avant garde de l'après-guerre il a su développer un style absolument personnel dont un rire s'est alors dégagé - déjà dans Aventures et Nouvelles Aventures qui est du théâtre musical très ironique, un peu à la Jarry. Son art est devenu à la fois un mariage original entre l'art populaire et l'art moderne et a d'une certaine manière décomplexé beaucoup de compositeurs. Notamment la reconquête d'un pulsation rythmique, une reconsidération de la consonance.

CFR : En cela Ligeti est singulier et complètement inclassable puisqu'on parlait d'étiquettes.

JEAN-MICHEL DENIZART : Vous retrouvez-vous dans les notions qu'évoquait Christophe? Et cela appelle une autre question qui est celle de l'étiquette. Toujours en rapport avec le ludisme, question en apparence simple : est-il possible d'étiqueter un compositeur?

RC : En France on aime beaucoup les étiquettes mais elles sont devenues très floues dans les années 70-80. Le terme d'avant-garde a commencé par être rejeté par les compositeurs d'avant garde eux-mêmes - je pense à Berio par exemple. 
Un nouveau terme-virus a vu le jour : celui de postmodernisme, un terme provenant de l'architecture et de la peinture, qui est arrivé avec le mouvement pop art mais surtout en musique avec de compositeurs pourtant très différents comme Arvo Pärt ou Philip Glass. Ligeti, tout en étant intéressé à la fois par Steve Reich et Terry Riley, des compositeurs typiquement postmodernes, ne se disait pas postmoderne pour autant. Mon avis est que l'on traverse une période plus "pacifique » et qu'on est dégagé de tout ce magma de termes anxiogènes. Peut-être sommes-nous dans un moment de post-post-modernisme!

CFR : On a bien sûr prévu de parler un petit peu d'actualité puisqu'en septembre 2014, vous faites créer Quai Ouest qui est un nouvel opéra d'après une pièce de Bernard-Marie Koltès à l'Opéra national du Rhin à Strasbourg puis à l'Opéra de Nuremberg en langue allemande. Pouvez-vous nous en dire quelques mots ? On a parlé de ludique et le texte de Quai Ouest à première vue n'est pas vraiment un texte humoristique, loin de là. L'humour et le tragique peuvent-ils être conciliables ? Comment a pu se passer ce travail pour vous ?

RC : La pièce a été créée en 1986 au théâtre Nanterre-Amandiers avec une mise de scène de Patrice Chéreau. J'ai relu plusieurs fois les indications de Koltès à la fin du livre aux éditions de Minuit: un texte intitulé "Pour mettre en scène Quai Ouest». Il parlait tout de suite d'une ironie que Chéreau avait peut-être occultée. Koltès avait écrit qu'il fallait «éliminer tout le tragique de cette histoire ». C'est un peu la démarche des vrais auteurs, d'être un peu contradictoires, de partir dans d'autres directions où du moins de les fantasmer, et présentement, d'aller de la tragédie vers ce besoin d'ironie. Cet humour koltésien, je l'ai alors cherché en composant mon opéra dans un langage dégagé de toute forme d'emphase et qui rejoint finalement l'idée d'une tragi-comédie.

Transformer une telle pièce de théâtre en un livret d'opéra était extrêmement difficile. A un moment donné il y a une souffrance du librettiste avec le compositeur car en définitive c'est l'œuvre du compositeur et sa propre vision doit s'imposer avant tout. Tous les collaborateurs de Kubrick ont exprimé une certaine amertume avec le sentiment d'avoir été manipulé.

JMD : Lors d'autres échanges, nous avions déjà abordé le thème de l'adaptation, et vous aviez évoqué l'idée que l'opéra devait contenir, je vous cite, «des impuretés ». Je trouvais la notion intéressante, pourriez-vous la développer aujourd'hui ?

RC : Pierre Boulez disait dans les années 50 qu'il fallait brûler les maisons d'opéra. Évidemment il a changé d'avis puisqu'il s'est mis à diriger des opéras dans des maisons d'opéra, et et même projeté l'écriture d'un opéra qui ne s'est jamais fait. Si l'on écrit une histoire de la musique contemporaine de l'après-guerre jusqu'à aujourd'hui, on pourrait peut être l'analyser uniquement à travers les productions d'opéras contemporains.

Or si on composait un opéra dans les années 50, on était un compositeur réactionnaire, néoclassique, vieillot. Chez les compositeurs d'avant-garde on ne parlait pas d'opéra mais de théâtre musical, voire même d'action musicale, d'antiopéra. Berio avait appelé son opéra Outis une "action musicale ». Ligeti a commencé à écrire du théâtre musical avec ses Nouvelles aventures, pour lui c'était de l'anti-opéra, puis, il a écrit avec son Grand Macabre de l'anti-anti opéra qui est devenu finalement un opéra tout court (rires). C'est pour vous dire que toutes ces étiquettes étaient une obsession chez beaucoup de compositeurs de cette génération. 
L'opéra depuis disons Monteverdi a toujours eu un rapport trouble avec l'art populaire et l'art expérimental. Ce mariage bizarre a existé dans les années 70 dans le cinéma italien par exemple : chez Pasolini, avec Théorème ou Salò, ou alors chez des auteurs de cinéma de genre comme Dario Argento avec ses giallos. Il y avait à cette époque une envie de suivre des codes narratifs académiques, populaires, mais d'être expérimental, dans le montage ou la photographie. Le cinéma a volé cela à la musique contemporaine qui elle a rejeté dans la forme de l'opéra contemporain ce dialogue ambigu du kitsch, du quotidien, de l'impur avec la sophistication de l'expérimental.

CFR : Comment aborde un compositeur aujourd'hui la musique de scène? Est-ce que justement le cinéma n'est pas devenu incontournable? En tout cas comment avez-vous envisagé ça?

RC : J'étais troublé que Kubrick parle de son film 2001, l'odyssée de l'espace comme d'une expérience non verbale et musicale. Quand on me demande quels sont les compositeurs d'aujourd'hui qui m'inspirent, je pense souvent à des réalisateurs de film comme David Lynch, ou Martin Scorsese. À quinze ans quand j'écoutais la bandeson de 2001, pour moi le compositeur était en réalité Kubrick et ils avaient ses assistants qui s'appelaient Richard Strauss, György Ligeti, Johann Strauss ou Aram Khachaturian. Scorsese dans son film Shutter Island a fait un montage d'après plusieurs musiques contemporaines pourtant très différentes et parfois certaines sont superposées. C'est un travail proche d'un compositeur d'opéra qui doit trouver une unité en partant d'éléments hétéroclites.

JMD : Pour revenir aux bandes originales et rester dans la thématique du Cinéma, je souhaitais vous demander quelles étaient les bandes originales qui vous avaient inspirées, mais vous avez anticipé cette question avec David Lynch et Martin Scorsese qui sont des réalisateurs, mais pensez-vous toutefois à certains compositeurs? Je pensais à Morricone notamment ou à des compositeurs de musique de film qui auraient pu vous inspirer dans votre travail de composition?

RC: Morricone, étonnamment, a commencé ses études à Rome, avec Goffredo Petrassi qui était le grand compositeur italien d'avant-garde, et il jouait dans le groupe d'improvisation Nuova Consonanza. Il a rencontré Scelsi et beaucoup de compositeurs contemporains notamment post-sériels puis il a mené une carrière de musicien de film en mêlant un art populaire avec un art expérimental. On retrouve ce balancement chez un compositeur italien stylistiquement bien éloigné : Luciano Berio avec ses Folk songs avec cette envie de régénérer dans sa propre musique d'avantgarde une couleur populaire, folklorique.

JMD : Lors de nos entrevues, nous avions évoqué Hans Zimmer et aussi d'autres compositeurs, postmodernes, dans le sens péjoratif du terme. Le mot PME est sorti. Est-ce que vous pourriez en parler un peu?

RC : Pour moi, le terme postmoderne en musique est un vilain mot, c'est clairement trier les poubelles de l'histoire musicale et la musique hollywoodien en est l'illustration. A la différence d'un Stravinsky qui reprend du Pergolèse ou ses contemporains pour écrire Pulcinella, c'est un acte de création avec de nouvelles idées inouïes d'écriture, avec une technique personnelle, écrit sans « assistant ».

CFR: II faut sans doute préciser pour l'auditoire que des compositeurs comme Hans Zimmer ou Danny Elfman ne composent pas toute leur musique seuls, ils composent peutêtre les mélodies de base et après ils ont toute une équipe d'assistants qui vont orchestrer et agencer les idées qu'ils auront émises, à la différence d'Howard Shore qui fait tout de 
bout en bout, ou même John Williams, même s'il verse parfois dans l'esthétique péjorative de ce terme postmoderne.

RC : Il y a une chose qu'on a jamais trop vue, c'est que justement dès les années $70, \mathrm{il}$ y a une récupération de l'art d'avant-garde européenne par Hollywood. Un auteur comme Penderecki a été pratiquement pillé. Sa musique a été utilisée chez Kubrick pour être ensuite copiée dans beaucoup de films hollywoodiens, surtout des films d'horreur ou fantastique où on entend très précisément des plagiats de De Natura Sonoris, Thrène ou de Polymorphia notamment.

1 Ecoute de Polymorphia de Krzysztof Penderecki

RC : On l'entend dans le film l'Exorciste d'ailleurs.

CFR : On peut peut-être continuer dans les extraits, on parlait du début de Shutter Island, avec la superposition de plusieurs compositeurs, en l'occurrence il s'agit de Ligeti et Marshall Ingram. Regardons le début du film.

2 Extrait de Shutter Island

RC : (commentaire de l'extrait) On entend ici cette intervalle de quarte augmentée provenant de l'œuvre Lontano de Ligeti et qui joue un rôle évidemment très dramatique. On en vient ensuite à Fog Tropes de Marshall Ingram. Là, Martin Scorsese travaille vraiment comme un compositeur, laisse entendre des bruits de vagues; ces cornes de brume un peu fausses et les cors proviennent de Fog Tropes mais Scorsese se les approprie comme les véritables sons de son film. On perçoit de moins en moins le bruit des moteurs du bateau alors qu'on aurait dû l'entendre dans la cabine, mais ça c'est une manipulation du réalisateur, c'est une manière de se transporter dans le cerveau du personnage qu'incarne Leonard DiCaprio. Ensuite vous entendez un orchestre symphonique qui imite nos cornes de brume: c'est un extrait de la Symphonie $n^{\circ} 3$ de Penderecki avec ses énormes octaves scandées. Avec le dialogue, cette mise en scène presque onirique, cette manière de composer les plans, le choix de la photographie, tout cela devient l'œuvre d'un seul auteur, Martin Scorsese en l'occurrence.

Il y a un terme que vous connaissez sûrement, employé par les historiens du cinéma, c'est le terme de diégétique. C'est la musique qui appartient strictement au film. Ça peut être une valse, ou un piano désaccordé dans un western, ou bien justement une corne de brume.

CFR : Ca me faisait penser à ll était une fois dans l'ouest.

RC : Si vous écoutez bien le début de ce film, Serge Leone créait toute une polyphonie composée de couinements des enseignes métalliques et un bruit d'éolienne; on entend ensuite un harmonica.

\section{Extrait de Il était une fois dans l'ouest}

RC : Les notes de l'harmonica sont en liaison avec le bruit d'éolienne du début, c'est vraiment la musique diégétique du film qui va devenir la musique du film.

CFR : Avant d'aborder David Lynch, je vais passer un dernier extrait, du film There will be blood.

RC : Au début de Il était une fois dans l'ouest, on perçoit le bruit du train, une sorte de brouhaha, de bourdon qui ressemble un peu à cette musique du début de There will be blood et je pense que le réalisateur Paul Thomas Anderson a pensé encore une fois à Kubrick avec 2001 : lorsque vous voyez le monolithe avec les singes, vous entendez 
cette même rumeur avec le Kyrie du Requiem de Ligeti. La musique est une sorte de trame harmonique étrange.

CFR : On est très loin de ce dont on disait tout à l'heure des compositeurs comme Hans Zimmer ou Danny Elfman, puisque là, il va s'inspirer de compositeurs plus modernes et pas seulement romantiques comme peuvent le faire Hans Zimmer ou Danny Elfman. Cette opposition appelle peut-être une question, complexe, qui est celle de la modernité. Qu'estce que la modernité ? Vous aviez évoqué lors de nos précédentes entrevues notamment une métaphore en référence au cinéma.

RC : Le compositeur du groupe Radiohead, Jonny Greenwood, est moderne au sens où il s'éloigne du postmodernisme; il n'est pas amnésique et il a même intégré l'atonalité d'un Penderecki ou d'un Ligeti des années 60 mais d'une manière très corporelle, très sensuelle.

Les compositeurs de musique contemporaine à la différence des écrivains, des peintres, des réalisateurs, des comédiens, des chorégraphes, semblent parfois avoir un souci avec leurs propres corps et ils semblent même parfois asexués. Je pense que dans cinquante ans, on pourra peut-être enfin parler des rapports de la sexualité des compositeurs d'aujourd'hui avec leurs propres créations comme on a pu le faire avec Picasso!

CFR : Il est vrai que la différence que je perçois en tant qu'auditeur et puis comme musicien, c'est que dans la musique de Johnny Greenwood il y a une vitalité qui provient de son expérience du rock, musique jouée sur scène pour laquelle il n'y a pas une possibilité autre que d'avoir un contact direct avec le public. Je pense que ça se retrouve.

Je voudrais revenir sur cette métaphore que vous aviez proposé dans une précédente entrevue à propos de Lynch et de Spielberg, avec cette différence qu'il y avait entre leurs deux cinémas et qui pouvait peut-être un peu résumer la différence qu'il y a entre Greenwood et Xenakis, cette idée de noyau qui irradie.

JMD : « Un film moderne part d'un noyau explicatif et irradie alors que chez un Lynch ou Kubrick, une force irradie, hypnotise, mais le noyau lui reste inaccessible. »

RC : C'est le cas aussi par exemple d'un film d'Alain Resnais qui invente la forme à chaque fois: on sent une force qui irradie, mais on ne comprend pas tout. Les premiers spectateurs du dernier film de Lynch Inland Empire ou de 2001 disaient « je ne comprends rien à l'histoire, de quoi parle-t-on? ». Avec Ligeti ou Boulez, la même question se pose. Mais ce qui fait la force de ces grands artistes c'est qu'il y a une sorte de noyau dur qu'on ne peut pas tout de suite déchiffrer et qui irradie, c'est ça la modernité. A l'inverse, Spielberg n'est pas forcément moderne, il est du moins plus traditionnel, et l'on voit tout de suite le noyau dur de ses films; tout le plaisir de voir ses films est d'apprécier sa virtuosité qui tourne autour de ce noyau.

JMD : On a beaucoup parlé de Stanley Kubrick et son cinéma-cerveau, on a beaucoup parlé de David Lynch et son cinéma schizophrénique et mystérieux. Juste pour ouvrir une parenthèse, on parlait tout à l'heure du caractère lacunaire des dialogues dans un Lost Highway avec à peu près 3300 mots, soit probablement deux fois moins que ce qui a dû être prononcé aujourd'hui. On comprend la confiance de David Lynch sur sa bande son, confiance pas anodine dans la mesure où il y a un travail intéressant de collaboration avec Angelo Badalamenti. Que vous évoque les bandes sons du cinéaste? Cette collaboration avec Angelo Badalamenti ou plus ponctuellement avec le leader de Nine Inch Nails, Trent Reznor.

RC : C'est un travail sur la lenteur qui est proche de Leone et bien sûr de Tarkovski (le compositeur Gérard Grisey nous en parlait souvent dans sa classe de composition). 
Je crois qu'il y a une œuvre cinématographique qui a marqué tous ces réalisateurs c'est le Mort à Venise de Visconti avec cette utilisation mémorable de l'adagietto de Mahler. Ce travail sur la lenteur chez David Lynch est vraiment obsessionnel où le temps narratif est complètement dilaté.

JMD : C'est vrai qu'il y a un certain fétichisme du bourdon et des nappes graves chez David Lynch.

RC : Il y a souvent un bourdonnement très grave, du vent, du souffle. Du vent comme chez Fellini au début de Huit et demi par exemple.

JMD : On avait évoqué le terme drone aussi dans d'autres communications pour ces fameuses nappes. Toujours pour rester avec Lynch, Christophe m'a parlé de votre rapport avec des musiques plus populaires. Je pense au fait que David Lynch utilise beaucoup d'œurres préexistantes à ses films, par exemple David Bowie, Rammstein ou encore Powermad qui sont composées avant et ré-exploitées à l'intérieur de son cinéma, quels sont les rapports que vous entretenez en tant que compositeur avec la musique plus populaire?

CFR : On a pu parler de Sigur Rós...

RC : Je ne connaissais pas ce groupe de rock islandais avant d'écrire mon opéra. C'est un peu anecdotique mais c'est un exemple intéressant. J'ai été frappé par leur utilisation de l'archet de violoncelle avec leurs guitares électriques.

Cette utilisation de l'archet ou de l'Ebow transforme leurs guitares en véritables violes de gambe électroniques. J'ai fréquemment utilisée cette manière d'écrire pour la guitare électrique et la basse dans mon opéra Quai Ouest comme une manière originale d'accompagner les voix.

JMD : Et si on vous laissait le mot de la fin ?

RC: Il y a un terme que l'on n'a pas utilisé, c'est le terme un peu pédant de métaphysique qui concerne Dario Argento, Lynch, Kubrick ou Ligeti. Pour moi l'opéra tend vers un art métaphysique, c'est-à-dire une recherche d'un autre monde, quelque chose de sacré, de mystérieux qui doit s'éloigner du bavardage.

\section{Questions du public}

Public : II a beaucoup été question de Cinéma, et plus que je ne l'aurais imaginé. Est-ce que vous avez composé pour des musiques de films?

RC : Oui mais très peu et il s'est posé pour moi le problème d'égo du réalisateur et de son compositeur. Au départ j'étais très intéressé par le travail pour le cinéma, mais je me suis de plus en plus aperçu que je voulais être le réalisateur, m'occuper du montage, du scénario. Si Scorsese a une très grande culture musicale c'est très peu le cas pour la plupart des réalisateurs - bien malheureusement.

CFR : C'est vrai que les extraits dont vous avez le plus parlé proviennent de réalisateurs qui ont une immense culture musicale.

$\mathbf{P}$ : Christophe a dit que vous aviez composé beaucoup de genres différents, et que vous écoutiez beaucoup de genres différents. Personnellement, dans quel genre vous sentezvous le plus à l'aise?

RC : En lui parlant de mon parcours, le compositeur Jean Prodromidès a tout de suite vu que j'étais proche d'un homme de théâtre, d'opéra. Il y a une narration et une 
dramaturgie musicale dans ma musique qui peut se connecter avec un livret d'opéra ou un texte. Si je compose sans texte, sans livret, c'est déjà une sorte d'opéra en soi.

JMD : On a parlé de la rencontre entre le compositeur et le réalisateur et tout à l'heure, quand j'ai évoqué Hans Zimmer, tout de suite Christophe a rebondi sur Danny Elfman. Alors peut-être un peu bêtement, j'osais imaginer que Danny Elfman, du moins dans son travail avec Tim Burton, n'était pas dans quelque chose de péjorativement postmoderne, du coup est-ce que vous pensez que le travail que réalise Danny Elfman avec Tim Burton, ou Danny Elfman avec x compositeurs dans une énergie un peu «PME », est-ce que vous pensez que c'est vraiment le même ou est-ce qu'il n'y aurait pas une certaine originalité dans le Danny Elfman que moi j'aime bien, à savoir celui qui travaille avec Burton?

RC : Votre question m'inspire que nous n'avons pas assez abordé la notion de kitsch. Le kitsch est lié à notre société de consommation, au marketing, à une constante 'aliénation de la société de masse. Mais le kitsch peut attirer de vrais créateurs d'une manière détournée. Cela me fait penser à une réflexion de Ligeti lorsqu'il écoutait en répétition une de ses dernières œuvres Sippam, Dobbal, Nàdihegedüvel, il dit à la chanteuse : « ce n'est pas trop kitsch, ma musique?». Mais il le disait avec l'envie que cela le soit un peu justement! C'est très intéressant cette envie d'être mauvais garçon, de faire preuve d'un certain mauvais genre...

P: Il a beaucoup été question de la musique des autres, est-il possible d'écouter la vôtre?

Ecoute de Cinq mélodies 4/5 de Régis Campo

$\mathbf{P}$ : On se faisait la remarque que c'était très scénique...

RC: Oui tout a fait, comme un ballet. Nous n'avons pas parlé du ballet aussi, mais c'est exact. Je voulais vous faire écouter aussi les Cris de Marseille enregistrés par l'ensemble Clément Janequin et Dominique Visse. Là c'est parfois la transcription de mélodies très populaires qui existent dans la rue, souvent de marchands; ma grandmère m'avait alors chanté plein d'airs de commerçants de rues qu'elle entendait toute petite dans les années 30 à Marseille. On peut ensuite écouter le Pic-vert écrit pour piccolo et piano.

\section{Ecoute de Pic-Vert de Régis Campo}

RC : La dernière pièce qu'on pourrait écouter c'est la dernière du disque Laterna Magica, le 2e mouvement de Mysterium Simplicitatis pour piano.

\section{Ecoute de Laterna Magica de Régis Campo}

RC : C'est une toute petite pièce pour jeune pianiste. C'est tout l'art de répéter en très peu de notes. Un ami japonais m'avait dit: "quand on répète deux fois, on copie ; quand on répète quatre ou cinq fois, c'est un hommage; mais quand on répète cent fois, c'est une prière ", j'avais beaucoup aimé cette phrase. C'est une technique musicale intéressante, il y a très peu de notes, je les répète et à un moment donné vous êtes complètement hypnotisé. Morton Feldam ou Ligeti le fait avec le 2e mouvement de son Musica Ricercata.

CFR : D'ailleurs, la pièce dont vous parlez se trouve dans Eyes wide shut.

RC : Il a construit tout un mouvement avec deux notes et l'idée, c'est d'être inventif à partir d'un élément très pauvre et très simple. Dans ce travail minimaliste, on arrive à créer un monde infini, ouvert, ce qui est paradoxal. 
$\mathbf{P}$ : On retrouve la même chose dans la danse. On va avoir des pièces dans lesquelles on va avoir toujours un nouveau mouvement à chaque fois, et des pièces où au contraire on va répéter un mouvement, le reprendre.

RC : Par moment il faut laisser vivre le vide à travers l'art. Le compositeur japonais Tōru Takemitsu travaille beaucoup avec un simple accord en résonance puis un silence. Ce silence peut se rappocher de la notion japonaise du $M a$, un terme intraduisible désignant une sorte de réceptacle où il n'y a rien, où on laisse résonner ce qu'il y a avant ou ce qui arrivera peut-être après. Le plein et le vide jouent ensemble. David Lynch justement est très influencé par cela dans un art cinématographique avec très peu de dialogues et une confiance dans des moments suspendus.

P : J'avais une question assez pragmatique, je ne connais vraiment rien à la manière de composer, mais je me dis qu'aujourd'hui avec les musiques assistées par ordinateur on ne compose pas de la même manière qu'au temps du crayon sur la partition. Alors comment composez-vous aujourd'hui ?

RC: Une des choses très importantes dans l'avant-garde d'après-guerre est son intérêt accru (avec le rythme) pour le timbre. Plus que pour la mélodie ou les hauteurs. Le terme de «musique assistées par ordinateur » veut tout dire et n'est pas très heureux. L'usage exclusif de l'ordinateur peut vite mener le compositeur vers une grande paresse auditive et un grand conformisme esthétique.

Le problème est de trop dépendre de l'outil informatique car il y a un moment où il faut toujours faire confiance à l'inconnu.

La fin de l'écriture de mon opéra Quai Ouest m'a rendu un peu inquiet car j'ai tenté beaucoup de choses nouvelles pour moi mais cette inquiétude est normale car elle m'a donné de l'adrénaline. Il y a quelque chose d'excitant dans ce lâcher-prise car l'on ne peut certes pas tout contrôler; il faut laisser vivre et respirer l'œuvre toute seule et faire confiance aux propositions créatives des interprètes eux-mêmes. 